\title{
A Cross-Study Biomarker Signature of Human Bronchial Epithelial Cells Infected with Respiratory Syncytial Virus
}

\author{
Luiz Gustavo Gardinassi \\ Department of Biochemistry and Immunology, Ribeirão Preto Medical School, University of São Paulo, \\ 14049-900 Ribeirão Preto, SP, Brazil \\ Correspondence should be addressed to Luiz Gustavo Gardinassi; gugard@gmail.com
}

Received 31 December 2015; Accepted 13 April 2016

Academic Editor: Jay C. Brown

Copyright (C) 2016 Luiz Gustavo Gardinassi. This is an open access article distributed under the Creative Commons Attribution License, which permits unrestricted use, distribution, and reproduction in any medium, provided the original work is properly cited.

\begin{abstract}
Respiratory syncytial virus (RSV) is a major cause of lower respiratory tract infections in children, elderly, and immunocompromised individuals. Despite of advances in diagnosis and treatment, biomarkers of RSV infection are still unclear. To understand the host response and propose signatures of RSV infection, previous studies evaluated the transcriptional profile of the human bronchial epithelial cell line-BEAS-2B-infected with different strains of this virus. However, the evolution of statistical methods and functional analysis together with the large amount of expression data provide opportunities to uncover novel biomarkers of inflammation and infections. In view of those facts publicly available microarray datasets from RSV-infected BEAS-2B cells were analyzed with linear model-based statistics and the platform for functional analysis InnateDB. The results from those analyses argue for the reevaluation of previously reported transcription patterns and biological pathways in BEAS-2B cell lines infected with RSV. Importantly, this study revealed a biosignature constituted by genes such as $A B C C 4, A R M C 8, B C L A F 1, E Z H 1, F A M 118 A, F A M 208 B$, FUS, HSPH1, KAZN, MAP3K2, N6AMT1, PRMT2, S100PBP, SERPINA1, TLK2, ZNF322, and ZNF337 which should be considered in the development of new molecular diagnosis tools.
\end{abstract}

\section{Introduction}

Respiratory syncytial virus (RSV) is a major etiologic agent causing acute lower respiratory infections that can progress to bronchiolitis and pneumonia in children, elderly, and immunocompromised individuals $[1,2]$. RSV outbreaks are influenced by virus diversity and evolution $[3,4]$, environmental factors [5], and host immunity [6].

The epithelium is the primary site for host-virus interface, where cells recognize pathogen-associated patterns on microbes through innate immunity receptors $[7,8]$. Indeed, epithelial cells constitute an important line of defense against RSV and other airborne pathogens [9]. They form a physical barrier and produce mucus to inhibit microbes from entering the body. Moreover, they express molecules with antimicrobial properties, as lysozyme, lactoferrin, collectins, and antimicrobial peptides [10]. Two human cell lines have been extensively used to understand the interaction between host and RSV, the alveolar epithelial cell, A549, and one from proximal airways, the bronchial epithelial cell, BEAS-2B.
Genome-wide microarrays are powerful tools to investigate host transcriptional response during infections in the pulmonary epithelium, including those induced by RSV $[11,12]$. Indeed, two studies evaluated the patterns of gene expression from BEAS-2B cell lines infected with RSV [10, 13]. However, it is intriguing that after $4 \mathrm{~h}$ of infection Huang and collaborators (2008) found that RSV-modulated genes were only associated with the neuroactive ligandreceptor interaction pathway [13]; in contrast, Mayer and collaborators (2007) identified that the same time of RSV infection of BEAS-2B cells induced transcriptional changes similar to those found for other respiratory pathogens as Pseudomonas aeruginosa [10]. In spite of differences, publicly available microarray data offers an interesting opportunity to reveal common features of RSV induced transcriptional profiles to understand the early response of BEAS-2B cell lines and extend the knowledge on biomarkers of acute infections with this virus. Therefore, those datasets were evaluated in a meta-analysis by fitting linear models for each array probe and Empirical Bayesian approach to detect 
transcriptional changes that revealed significant associations with unreported pathways. Of importance, this strategy also rendered a biomarker signature of BEAS-2B cell lines infected with RSV that can be useful for the design of molecular diagnosis tools.

\section{Materials and Methods}

The datasets GSE3397 and GSE6802 were obtained from GEO database (http://www.ncbi.nlm.nih.gov/), which compared BEAS-2B cells infected with RSV with control experiments. Only arrays in which cells were infected with RSV for $4 \mathrm{~h}$ were selected for further analysis. Raw data were processed using the R Language and Environment for Statistical Computing (R) 3.2.0 [14] and Bioconductor 3.1 [15]. The affy package for $\mathrm{R}$ [16] was used to perform quality control when applicable. Data was $\log _{2}$ transformed and quantile normalization was applied for dataset GSE3397 due the absence of CEL files. The dataset GSE6802 was already RMA normalized. Batch effects were corrected with Combat( ) function [17] of sva package for $\mathrm{R}$ [18]. Expression data were weighted with the arrayWeights() function from limma package for $\mathrm{R}$ [19]. Differential gene expression was also evaluated with limma package for R [19], whereby differentially expressed genes (DEGs) were identified by a false discovery rate (FDR) $<0.05$. Hierarchical clustering was performed with Euclidian distance for metric calculations and the complete linkage method, which were displayed as heatmaps drawn with gplots package for R [20]. Pathway analyses were performed with the online platform for functional analysis InnateDB [21] and significant pathway overrepresentation was computed with hypergeometrical distribution and BenjaminiHochberg correction for multiple comparisons. Significantly enriched pathways were determined by a $P$ value $<0.05$ and FDR $<0.1$.

\section{Results and Discussion}

3.1. Dataset Selection and Preprocessing Analysis. To define a robust transcriptional signature of BEAS-2B acutely infected with RSV, two publicly available datasets, GSE3397 and GSE6802, were used to conduct a meta-analysis from which data were extracted for BEAS-2B cells infected with RSV for $4 \mathrm{~h}$ and controls. First, background subtracted expression data from GSE3397 (Figure 1(a)) were preprocessed and normalized (Figure 1(b)). However, in a first attempt to conduct differential gene expression analysis using limma [19], there were no statistically significant differences in gene expression. Therefore, principal component analysis (PCA) was used to evaluate the expression profiles of each array and, except for arrays named here Control 2 and RSV2, the consistent pattern of clustering in Figure 1(c) suggests a batch effect. After normalization, this effect was even more evident (Figure $1(\mathrm{~d})$ ), which led to the speculation that Huang and collaborators (2008) [13] analyzed only three microarray experiments from this dataset based on the assumption that differences found for those microarrays were due to failures in experimental procedures; however they did not consider or correct for batch effects. In view of those facts, the datasets were adjusted with Combat function for $\mathrm{R}$, which removed such effects from GSE3397 expression data (Figure 1(e)). Batch correction of GSE3397 did not change the profiles of arrays Control2 and RSV2; nevertheless, those arrays were included in further analysis because the variation observed in this experiment could have a substantial impact over the final result. Even adverse experimental variations that may change the overall expression patterns of a dataset could be useful to power up the identification of genes that are robustly modulated in BEAS-2B cells infected with RSV. The expression dataset GSE6802 (Figure 1(f)) was also included in the analysis. PCA from expression data extracted from GEO demonstrates that most of the variability between the arrays is explained (76.6\%) by the infection with RSV, as the standardized PC1 separates RSV-infected from control arrays (Figure $1(\mathrm{~g})$ ), whereas standardized PC2 (11.4\%) separates one pair of arrays (RSV_3 and ctrl2) and, although these arrays are supposedly from different batches, clustering features of this axis also suggested a batch effect (Figure $1(\mathrm{~g})$ ). $\log _{2}$ transformation of data impacted the profile of array RSV_1 however did not change the profiles from RSV_3 and ctrl_2 (Figure 1(h)). Combat ( ) function was also applied to the expression dataset GSE6802; however, PCA shows that the adjustment did not to improve further clustering between specific arrays (Supplementary Figure 1; see Supplementary Material available online at http://dx.doi.org/10.1155/2016/3605302). In view of that, downstream analyses were carried out with normalized $\log _{2}$ transformed data.

3.2. Differential Gene Expression. Next, linear model-based statistical analyses with a FDR $<0.05$ were conducted to identify differentially expressed genes (DEGs). The dataset GSE3397 exhibited ninety-four DEGs (Figure 2(a) and Table 1). Those genes are highly discordant from DEGs previously reported by Huang and collaborators (2008) [13], which identified 277 DEGs based on different statistical analysis and assumptions. Fifty genes were downregulated and fortyfour were upregulated (Table 1). The differences found in this study might reflect the inclusion of all microarray experiments from controls and $4 \mathrm{~h}$ after RSV infection; exclusion of expression data from $24 \mathrm{~h}$ after RSV infection; distinct preprocessing approaches as normalizing method and batch effect correction; and the assessment of statistical significance with a linear model-based method and corrected $P$ values. In contrast, 1965 DEGs were identified for the dataset GSE6802. The top hundred DEGs ranked by fold changes (Figure 2(b) and Table 2) included genes such as JUNB, KLF4, CXCL1, CXCL2, and IL6, which are in agreement with those reported by Mayer and collaborators (2007) [10]. Several factors should account for the notable differences in expression analysis from both datasets. First, different RSV strains were used to stimulate BEAS-2B cells. Second, experimental conditions of controls were also different, as control experiments from GSE3397 were incubated with vehicle (not specified) and those from GSE6802 were not stimulated. Third, despite both datasets being generated with affymetrix microarray platform, those include distinct versions, HU133 plus 2.0 for GSE3397 and HU133A 2.0 for GSE6802. 
TABLE 1: Differentially expressed genes identified in dataset GSE3397.

\begin{tabular}{|c|c|c|c|c|}
\hline ProbeID & Gene symbol & Gene name & $\log _{2}$ fold change & FDR \\
\hline 1560754_at & CMTM7 & CKLF like MARVEL transmembrane domain containing 7 & $-1,54756$ & 0,017104 \\
\hline 239439_at & AFF4 & AF4/FMR2 family member 4 & $-1,53581$ & 0,023832 \\
\hline 238929_at & SRSF8 & Serine/arginine-rich splicing factor 8 & $-1,51887$ & 0,018433 \\
\hline 223142_s_at & UCK1 & Uridine-cytidine kinase 1 & $-1,47939$ & 0,017104 \\
\hline 242636_at & PRCP & Prolylcarboxypeptidase & $-1,45095$ & 0,034358 \\
\hline 228007_at & CEP85L & Centrosomal protein $85 \mathrm{kDa}$-like & $-1,4103$ & 0,017104 \\
\hline 235573_at & HSPH1 & Heat shock protein family $\mathrm{H}(\mathrm{Hsp} 110)$ member 1 & $-1,39959$ & 0,0371 \\
\hline 228391_at & CYP4V2 & Cytochrome P450 family 4 subfamily V member 2 & $-1,38799$ & 0,01671 \\
\hline 219376_at & ZNF322 & Zinc finger protein 322 & $-1,3491$ & 0,046761 \\
\hline 1553689_s_at & METTL6 & Methyltransferase like 6 & $-1,34723$ & 0,017104 \\
\hline 242837_at & SRSF4 & Serine/arginine-rich splicing factor 4 & $-1,34071$ & 0,044693 \\
\hline 237215_s_at & TFRC & Transferrin receptor & $-1,32685$ & 0,017104 \\
\hline 208819_at & RAB8A & RAB8A, member RAS oncogene family & $-1,32593$ & 0,042264 \\
\hline 236665_at & CCDC18 & Coiled-coil domain containing 18 & $-1,31494$ & 0,034201 \\
\hline 206147_x_at & SCML2 & Sex comb on midleg-like 2 (Drosophila) & $-1,30586$ & 0,016454 \\
\hline 229325_at & ZZZ3 & Zinc finger ZZ-type containing 3 & $-1,30495$ & 0,017104 \\
\hline 1565716_at & FUS & FUS RNA binding protein & $-1,29415$ & 0,049505 \\
\hline 205062_X_at & ARID4A & AT-rich interaction domain $4 \mathrm{~A}$ & $-1,28877$ & 0,033039 \\
\hline 1552312_a_at & MFAP3 & Microfibrillar associated protein 3 & $-1,28521$ & 0,046511 \\
\hline 223223_at & ARV1 & ARV1 homolog, fatty acid homeostasis modulator & $-1,27987$ & 0,023832 \\
\hline 232001_at & PRKCQ-AS1 & PRKCQ antisense RNA 1 & $-1,27987$ & 0,035983 \\
\hline 233195_at & DNAI1 & Dynein axonemal intermediate chain 1 & $-1,25963$ & 0,047083 \\
\hline 219094_at & ARMC8 & Armadillo repeat containing 8 & $-1,25527$ & 0,043392 \\
\hline 235232_at & GMEB1 & Glucocorticoid modulatory element binding protein 1 & $-1,2492$ & 0,046511 \\
\hline 218643_s_at & CRIPT & CXXC repeat containing interactor of PDZ3 domain & $-1,24229$ & 0,0371 \\
\hline 1566851_at & TRIM42 & Tripartite motif containing 42 & $-1,24057$ & 0,042149 \\
\hline 221821_s_at & KANSL2 & KAT8 regulatory NSL complex subunit 2 & $-1,23799$ & 0,017104 \\
\hline 244115_at & FAM126A & Family with sequence similarity 126 member A & $-1,23114$ & 0,033039 \\
\hline 215541_s_at & DIAPH1 & Diaphanous related formin 1 & $-1,22774$ & 0,033039 \\
\hline 203196_at & $\mathrm{ABCC} 4$ & ATP binding cassette subfamily $\mathrm{C}$ member 4 & $-1,22519$ & 0,033039 \\
\hline 225024_at & RPRD1B & Regulation of nuclear pre-mRNA domain containing $1 \mathrm{~B}$ & $-1,22264$ & 0,043765 \\
\hline 37860_at & ZNF337 & Zinc finger protein 337 & $-1,22095$ & 0,023832 \\
\hline 212997_s_at & TLK2 & Tousled like kinase 2 & $-1,21841$ & 0,04814 \\
\hline 225690_at & CDK12 & Cyclin-dependent kinase 12 & $-1,21083$ & 0,0371 \\
\hline 232103_at & BPNT1 & $3^{\prime}\left(2^{\prime}\right), 5^{\prime}$-Bisphosphate nucleotidase 1 & $-1,20748$ & 0,0371 \\
\hline 224848_at & CDK6 & Cyclin-dependent kinase 6 & $-1,20247$ & 0,0371 \\
\hline 214962_s_at & NUP160 & Nucleoporin 160 kDa & $-1,20247$ & 0,046319 \\
\hline 219629_at & FAM118A & Family with sequence similarity 118 member A & $-1,19831$ & 0,028374 \\
\hline 212290_at & SLC7A1 & Solute carrier family 7 member 1 & $-1,19748$ & 0,042264 \\
\hline 227187_at & CBLL1 & Cbl proto-oncogene like 1, E3 ubiquitin protein ligase & $-1,19582$ & 0,030047 \\
\hline 233208_x_at & CPSF2 & Cleavage and polyadenylation specific factor 2 & $-1,19334$ & 0,046319 \\
\hline 230566_at & MORC2-AS1 & MORC2 antisense RNA 1 & $-1,17691$ & 0,0371 \\
\hline 238795_at & FAM208B & Family with sequence similarity 208 member B & $-1,17609$ & 0,0371 \\
\hline 204980_at & CLOCK & Clock circadian regulator & $-1,17283$ & 0,0371 \\
\hline 238653_at & LRIG2 & Leucine-rich repeats and immunoglobulin like domains 2 & $-1,17202$ & 0,048527 \\
\hline 229939_at & ENDOV & Endonuclease V & $-1,16878$ & 0,041349 \\
\hline 218185_s_at & ARMC1 & Armadillo repeat containing 1 & $-1,16151$ & 0,046319 \\
\hline 201083_s_at & BCLAF1 & BCL2 associated transcription factor 1 & $-1,15509$ & 0,049505 \\
\hline
\end{tabular}


TABle 1: Continued.

\begin{tabular}{|c|c|c|c|c|}
\hline ProbeID & Gene symbol & Gene name & $\log _{2}$ fold change & FDR \\
\hline 227840_at & C2orf76 & Chromosome 2 open reading frame 76 & $-1,15109$ & 0,042264 \\
\hline 201686_x_at & API5 & Apoptosis inhibitor 5 & $-1,14076$ & 0,046761 \\
\hline 221699_s_at & DDX50 & DEAD-box helicase 50 & 1,140764 & 0,046511 \\
\hline 1556178_x_at & TAF8 & TATA-box binding protein associated factor 8 & 1,159096 & 0,034358 \\
\hline 205623_at & ALDH3A1 & Aldehyde dehydrogenase 3 family member Al & 1,163927 & 0,049505 \\
\hline 212495_at & KDM4B & Lysine demethylase $4 \mathrm{~B}$ & 1,193336 & 0,044693 \\
\hline 1569057_s_at & MIA3 & Melanoma inhibitory activity family member 3 & 1,193336 & 0,047866 \\
\hline 222494_at & FOXN3 & Forkhead box N3 & 1,19582 & 0,048527 \\
\hline 223311_s_at & MTA3 & Metastasis associated 1 family member 3 & 1,19582 & 0,041439 \\
\hline 215424_s_at & SNW1 & SNW domain containing 1 & 1,196649 & 0,049505 \\
\hline 213478_at & KAZN & Kazrin, periplakin interacting protein & 1,19914 & 0,025143 \\
\hline 227864_s_at & MVB12A & Multivesicular body subunit $12 \mathrm{~A}$ & 1,201636 & 0,030287 \\
\hline 228674_s_at & EML4 & Echinoderm microtubule associated protein like 4 & 1,204137 & 0,040345 \\
\hline 224196_x_at & DPH5 & Diphthamide biosynthesis 5 & 1,205808 & 0,025143 \\
\hline 224652_at & CCNY & Cyclin Y & 1,207481 & 0,046761 \\
\hline 212968_at & RFNG & RFNG O-fucosylpeptide 3-beta- $\mathrm{N}$-acetylglucosaminyltransferase & 1,211673 & 0,0371 \\
\hline 1555486_a_at & PRR5L & Proline rich 5 like & 1,212513 & 0,017104 \\
\hline 232837_at & KIF13A & Kinesin family member $13 \mathrm{~A}$ & 1,214195 & 0,042264 \\
\hline 224320_s_at & MCM8 & $\begin{array}{l}\text { Minichromosome maintenance } 8 \text { homologous recombination repair } \\
\text { factor }\end{array}$ & 1,217566 & 0,033039 \\
\hline 230131_x_at & ARSD & Arylsulfatase D & 1,221793 & 0,0371 \\
\hline 218225_at & ECSIT & ECSIT signalling integrator & 1,224336 & 0,034358 \\
\hline 222610_s_at & $\mathrm{S} 100 \mathrm{PBP}$ & S100P binding protein & 1,226885 & 0,030047 \\
\hline 32259_at & EZH1 & Enhancer of zeste 1 polycomb repressive complex 2 subunit & 1,229439 & 0,0371 \\
\hline 203854_at & CFI & Complement factor I & 1,232852 & 0,042264 \\
\hline 221600_s_at & AAMDC & Adipogenesis associated, Mth938 domain containing & 1,260503 & 0,0371 \\
\hline 209558_s_at & HIP1R & Huntingtin interacting protein 1 related & 1,263127 & 0,042264 \\
\hline 224814_at & DPP7 & Dipeptidyl peptidase 7 & 1,26488 & 0,016454 \\
\hline 232280_at & SLC25A29 & Solute carrier family 25 member 29 & 1,277214 & 0,030047 \\
\hline 228424_at & NAALADL1 & N-Acetylated alpha-linked acidic dipeptidase-like 1 & 1,286989 & 0,042264 \\
\hline 203409_at & DDB2 & Damage specific DNA binding protein 2 & 1,288775 & 0,023832 \\
\hline 229975_at & BMPR1B & Bone morphogenetic protein receptor type $1 \mathrm{~B}$ & 1,297739 & 0,034358 \\
\hline 227073_at & MAP3K2 & Mitogen-activated protein kinase kinase kinase 2 & 1,297739 & 0,017104 \\
\hline 225347_at & ARL8A & ADP ribosylation factor like GTPase $8 \mathrm{~A}$ & 1,298639 & 0,02672 \\
\hline 221774_x_at & SUPT20H & SPT20 homolog, SAGA complex component & 1,308578 & 0,016454 \\
\hline 223679_at & CTNNB1 & Catenin beta 1 & 1,318594 & 0,018043 \\
\hline 227679_at & HDAC11 & Histone deacetylase 11 & 1,328686 & 0,044693 \\
\hline 220020_at & XPNPEP3 & X-Prolyl aminopeptidase 3, mitochondrial & 1,342573 & 0,031097 \\
\hline 203199_s_at & MTRR & 5-Methyltetrahydrofolate-homocysteine methyltransferase reductase & 1,360371 & 0,017104 \\
\hline 228722_at & PRMT2 & Protein arginine methyltransferase 2 & 1,370783 & 0,016454 \\
\hline 228951_at & SLC38A7 & Solute carrier family 38 member 7 & 1,431969 & 0,016454 \\
\hline 217529_at & ORAI2 & ORAI calcium release-activated calcium modulator 2 & 1,453973 & 0,043775 \\
\hline 220311_at & N6AMT1 & N-6 adenine-specific DNA methyltransferase 1 (putative) & 1,460032 & 0,017104 \\
\hline 213402_at & ZNF787 & Zinc finger protein 787 & 1,469169 & 0,017104 \\
\hline 226055_at & ARRDC2 & Arrestin domain containing 2 & 1,477338 & 0,017104 \\
\hline 219756_s_at & POF1B & Premature ovarian failure, $1 \mathrm{~B}$ & 1,580083 & 0,016454 \\
\hline 202833_s_at & SERPINA1 & $\begin{array}{l}\text { Serpin peptidase inhibitor, clade A (alpha-1 antiproteinase, antitrypsin), } \\
\text { and member } 1\end{array}$ & 2,488023 & 0,0371 \\
\hline
\end{tabular}


TABLE 2: Top hundred differentially expressed genes identified in dataset GSE6802.

\begin{tabular}{|c|c|c|c|c|}
\hline ProbeID & Gene symbol & Gene name & $\log _{2}$ fold change & FDR \\
\hline 212615_at & CHD9 & Chromodomain helicase DNA binding protein 9 & $-3,69609$ & 0,00131 \\
\hline 221840_at & PTPRE & Protein tyrosine phosphatase, receptor type E & $-3,56524$ & 0,000195 \\
\hline 220817_at & TRPC4 & Transient receptor potential cation channel subfamily $\mathrm{C}$ member 4 & $-3,39168$ & 0,001582 \\
\hline 221703_at & BRIP1 & BRCA1 interacting protein C-terminal helicase 1 & $-2,88786$ & 0,021463 \\
\hline 207012_at & MMP16 & Matrix metallopeptidase 16 & $-2,82647$ & 0,000119 \\
\hline 219494_at & RAD54B & RAD54 homolog B (S. cerevisiae) & $-2,81279$ & 0,000177 \\
\hline 207034_s_at & GLI2 & GLI family zinc finger 2 & $-2,79723$ & 0,005157 \\
\hline 203518_at & LYST & Lysosomal trafficking regulator & $-2,75872$ & $5,90 E-05$ \\
\hline 205282_at & LRP8 & LDL receptor related protein 8 & $-2,7549$ & 0,000311 \\
\hline 214440_at & NAT1 & $\mathrm{N}$-Acetyltransferase 1 (arylamine $\mathrm{N}$-acetyltransferase) & $-2,68515$ & 0,001777 \\
\hline 219627_at & ZNF767P & Zinc finger family member 767 , pseudogene & $-2,67957$ & 0,00024 \\
\hline 218984_at & PUS7 & Pseudouridylate synthase 7 (putative) & $-2,67586$ & 0,001308 \\
\hline 206554_x_at & SETMAR & SET domain and mariner transposase fusion gene & $-2,63536$ & 0,002432 \\
\hline 219779_at & ZFHX4 & Zinc finger homeobox 4 & $-2,62624$ & 0,001411 \\
\hline 213103_at & STARD13 & StAR related lipid transfer domain containing 13 & $-2,57219$ & 0,002525 \\
\hline 210138_at & RGS20 & Regulator of G-protein signaling 20 & $-2,55974$ & 0,000415 \\
\hline 204291_at & ZNF518A & Zinc finger protein $518 \mathrm{~A}$ & $-2,54383$ & $9,70 E-05$ \\
\hline 204651_at & NRF1 & Nuclear respiratory factor 1 & $-2,49147$ & 0,003659 \\
\hline 205408_at & MLLT10 & Myeloid/lymphoid or mixed-lineage leukemia; translocated to, 10 & $-2,48975$ & $5,10 E-05$ \\
\hline 219581_at & TSEN2 & tRNA splicing endonuclease subunit 2 & $-2,45377$ & 0,001774 \\
\hline 218242_S_at & SUV420H1 & Lysine methyltransferase $5 \mathrm{~B}$ & $-2,44698$ & 0,000754 \\
\hline 203242_S_at & PDLIM5 & PDZ and LIM domain 5 & $-2,43851$ & 0,001699 \\
\hline 203868_s_at & VCAM1 & Vascular cell adhesion molecule 1 & $-2,43513$ & 0,000761 \\
\hline 220206_at & ZMYM1 & Zinc finger MYM-type containing 1 & $-2,36362$ & 0,008439 \\
\hline 207616_s_at & TANK & TRAF family member associated NFKB activator & $-2,34567$ & 0,000424 \\
\hline 218303_x_at & KRCC1 & Lysine-rich coiled-coil 1 & $-2,34567$ & 0,003187 \\
\hline 218490_s_at & ZNF302 & Zinc finger protein 302 & $-2,32785$ & 0,001816 \\
\hline 206876_at & SIM1 & Single-minded family bHLH transcription factor 1 & $-2,32624$ & 0,001681 \\
\hline 219128_at & C2orf42 & Chromosome 2 open reading frame 42 & $-2,28628$ & 0,002926 \\
\hline 212861_at & MFSD5 & Major facilitator superfamily domain containing 5 & $-2,27048$ & 0,000823 \\
\hline 218653_at & SLC25A15 & Solute carrier family 25 member 15 & $-2,25636$ & 0,000562 \\
\hline 206943_at & TGFBR1 & Transforming growth factor beta receptor I & $-2,24856$ & 0,025349 \\
\hline 201995_at & EXT1 & Exostosin glycosyltransferase 1 & $-2,247$ & 0,000421 \\
\hline 221430_s_at & RNF146 & Ring finger protein 146 & $-2,23457$ & 0,001084 \\
\hline 212286_at & ANKRD12 & Ankyrin repeat domain 12 & $-2,2253$ & 0,00029 \\
\hline 219544_at & BORA & Bora, aurora kinase A activator & $-2,21914$ & 0,000333 \\
\hline 210455_at & R3HCC1L & $\mathrm{R} 3 \mathrm{H}$ domain and coiled-coil containing 1 like & $-2,2176$ & 0,0039 \\
\hline 219459_at & POLR3B & Polymerase (RNA) III subunit B & $-2,2176$ & 0,000832 \\
\hline 219078_at & GPATCH2 & G-patch domain containing 2 & $-2,19923$ & 0,000723 \\
\hline 204547_at & RAB40B & RAB40B, member RAS oncogene family & $-2,17648$ & 0,001741 \\
\hline 209760_at & KIAA0922 & KIAA0922 & $-2,17347$ & 0,001048 \\
\hline 218791_s_at & KATNBL1 & Katanin regulatory subunit B1 like 1 & $-2,17347$ & 0,001187 \\
\hline 205173_x_at & CD58 & CD58 molecule & $-2,17196$ & 0,00022 \\
\hline 204352_at & TRAF5 & TNF receptor associated factor 5 & $-2,16895$ & 0,002659 \\
\hline 212441_at & KIAA0232 & KIAA0232 & $-2,16595$ & 0,006084 \\
\hline 204236_at & FLI1 & Fli-1 proto-oncogene, ETS transcription factor & $-2,15397$ & 0,005141 \\
\hline 203072_at & MYO1E & Myosin IE & $-2,15248$ & 0,000154 \\
\hline 219904_at & ZSCAN5A & Zinc finger and SCAN domain containing $5 \mathrm{~A}$ & $-2,14801$ & 0,00144 \\
\hline 219133_at & OXSM & 3-Oxoacyl-ACP synthase, mitochondrial & $-2,12285$ & 0,002424 \\
\hline 205798_at & IL7R & Interleukin 7 receptor & $-2,11257$ & 0,00506 \\
\hline
\end{tabular}


TABLE 2: Continued.

\begin{tabular}{|c|c|c|c|c|}
\hline ProbeID & Gene symbol & Gene name & $\log _{2}$ fold change & FDR \\
\hline 205476_at & CCL20 & C-C motif chemokine ligand 20 & 4,613942 & $9,50 E-05$ \\
\hline 213497_at & ABTB2 & Ankyrin repeat and BTB domain containing 2 & 4,623547 & $1,40 E-05$ \\
\hline 219179_at & DACT1 & Dishevelled-binding antagonist of beta-catenin 1 & 4,642816 & $9,00 E-06$ \\
\hline 219228_at & ZNF331 & Zinc finger protein 331 & 4,723971 & $6,00 E-06$ \\
\hline 213139_at & SNAI2 & Snail family zinc finger 2 & 4,76673 & $1,40 E-05$ \\
\hline 218177_at & CHMP1B & Charged multivesicular body protein $1 \mathrm{~B}$ & 4,806544 & $1,00 E-05$ \\
\hline 203304_at & BAMBI & BMP and activin membrane-bound inhibitor & 4,826576 & $3,00 E-06$ \\
\hline 201631_s_at & IER3 & Immediate early response 3 & 4,833271 & $3,00 E-06$ \\
\hline 218559_s_at & MAFB & v-maf avian musculoaponeurotic fibrosarcoma oncogene homolog B & 4,870264 & 0,000468 \\
\hline 220266_s_at & KLF4 & Kruppel-like factor 4 (gut) & 4,890561 & 0,00022 \\
\hline 209211_at & KLF5 & Kruppel-like factor 5 (intestinal) & 4,924578 & 0,002036 \\
\hline 209681_at & SLC19A2 & Solute carrier family 19 member 2 & 4,927992 & $5,90 E-05$ \\
\hline 205266_at & LIF & Leukemia inhibitory factor & 4,955395 & $2,20 E-05$ \\
\hline 204790_at & SMAD7 & SMAD family member 7 & 5,073566 & 0,000283 \\
\hline 221667_s_at & HSPB8 & Heat shock protein family B (small) member 8 & 5,422657 & $2,90 E-05$ \\
\hline 212665_at & TIPARP & TCDD-inducible poly(ADP-ribose) polymerase & 5,525098 & $1,00 E-05$ \\
\hline 202935_s_at & SOX9 & SRY-box 9 & 5,971114 & $3,30 E-05$ \\
\hline 202023_at & EFNA1 & Ephrin-A1 & 6,164569 & $3,30 E-05$ \\
\hline 202393_s_at & KLF10 & Kruppel-like factor 10 & 6,194552 & 0,000195 \\
\hline 213146_at & KDM6B & Lysine demethylase 6B & 6,203146 & $1,90 E-05$ \\
\hline 205193_at & MAFF & v-maf avian musculoaponeurotic fibrosarcoma oncogene homolog F & 6,2941 & $2,00 E-06$ \\
\hline 209457_at & DUSP5 & Dual specificity phosphatase 5 & 6,639157 & $1,30 E-05$ \\
\hline 206029_at & ANKRD1 & Ankyrin repeat domain 1 & 6,65759 & 0,008591 \\
\hline 209283_at & CRYAB & Crystallin alpha B & 6,703897 & 0,000118 \\
\hline 201693_s_at & EGR1 & Early growth response 1 & 7,056731 & $4,10 E-05$ \\
\hline 212099_at & RHOB & ras homolog family member B & 7,300524 & 0,000406 \\
\hline 219682_s_at & TBX3 & T-box 3 & 7,722136 & $5,80 E-05$ \\
\hline 201473_at & JUNB & jun B proto-oncogene & 8,322402 & $7,00 E-06$ \\
\hline 200664_s_at & DNAJB1 & DnaJ heat shock protein family (Hsp40) member B1 & 8,586082 & $2,00 E-05$ \\
\hline 205828_at & MMP3 & Matrix metallopeptidase 3 & 8,711976 & $1,90 E-05$ \\
\hline 201169_s_at & BHLHE40 & Basic helix-loop-helix family member e 40 & 8,870405 & 0,00011 \\
\hline 203665_at & HMOX1 & Heme oxygenase 1 & 9,32433 & 0,000544 \\
\hline 202643_s_at & TNFAIP3 & TNF alpha induced protein 3 & 9,573192 & $2,50 E-05$ \\
\hline 205207_at & IL6 & Interleukin 6 & 10,18236 & $3,00 E-06$ \\
\hline 202388_at & RGS2 & Regulator of G-protein signaling 2 & 10,25318 & $1,40 E-05$ \\
\hline 204472_at & GEM & GTP binding protein overexpressed in skeletal muscle & 10,8003 & $1,00 E-06$ \\
\hline 202149_at & NEDD9 & Neural precursor cell expressed, developmentally down-regulated 9 & 11,06553 & $2,50 E-05$ \\
\hline 219480_at & SNAI1 & Snail family zinc finger 1 & 11,70457 & $2,00 E-06$ \\
\hline 218839_at & HEY1 & hes related family bHLH transcription factor with YRPW motif 1 & 12,07541 & $6,00 E-06$ \\
\hline 206115_at & EGR3 & Early growth response 3 & 14,19194 & $1,20 E-05$ \\
\hline 204470_at & CXCL1 & $\mathrm{C}-\mathrm{X}-\mathrm{C}$ motif chemokine ligand 1 & 17,61827 & $2,00 E-06$ \\
\hline 204621_s_at & NR4A2 & Nuclear receptor subfamily 4 group A member 2 & 18,77837 & 0 \\
\hline 209774_x_at & CXCL2 & $\mathrm{C}-\mathrm{X}-\mathrm{C}$ motif chemokine ligand 2 & 19,02731 & $9,00 E-06$ \\
\hline 202859_x_at & CXCL8 & $\mathrm{C}-\mathrm{X}-\mathrm{C}$ motif chemokine ligand 8 & 19,89039 & $1,00 E-06$ \\
\hline 202340_x_at & NR4A1 & Nuclear receptor subfamily 4 group A member 1 & 20,74943 & $1,00 E-06$ \\
\hline 209189_at & FOS & FBJ murine osteosarcoma viral oncogene homolog & 23,36051 & $1,00 E-06$ \\
\hline 202672_s_at & ATF3 & Activating transcription factor 3 & 24,18432 & 0 \\
\hline 202768_at & FOSB & FBJ murine osteosarcoma viral oncogene homolog B & 32,92245 & 0 \\
\hline 207978_s_at & NR4A3 & Nuclear receptor subfamily 4 group A member 3 & 43,80428 & $1,00 E-06$ \\
\hline 117_at & HSPA6 & Heat shock protein family A (Hsp70) member 6 & 90,82389 & 0 \\
\hline
\end{tabular}




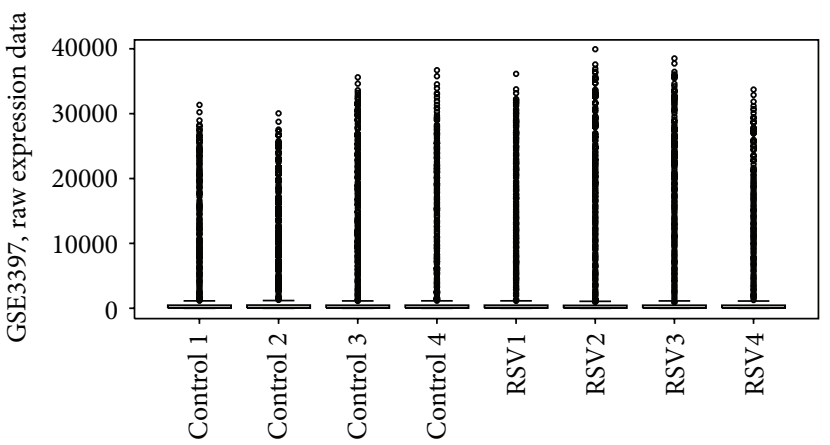

(a)

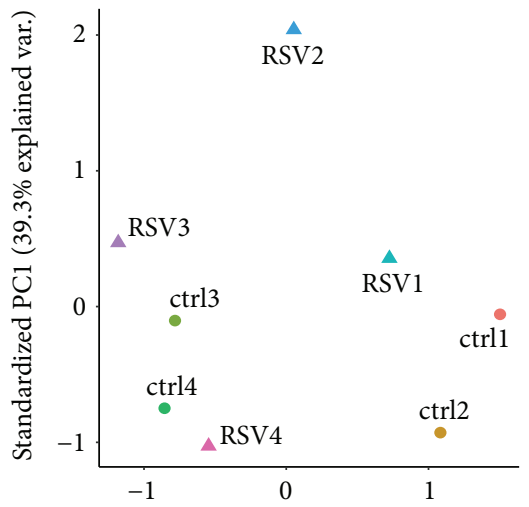

Standardized PC2 (29.9\% explained var.)

(c)

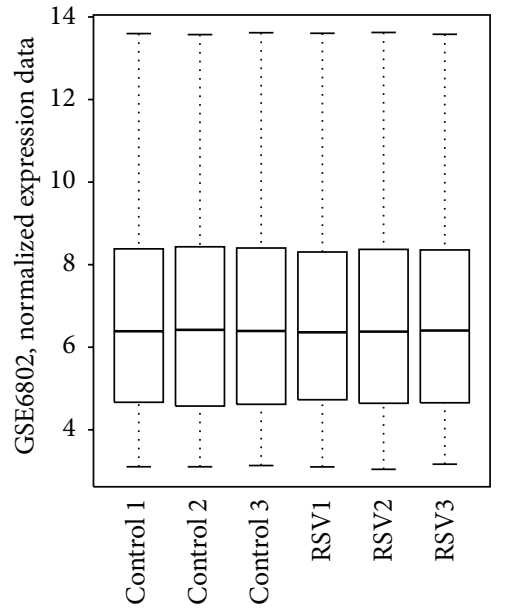

(f)

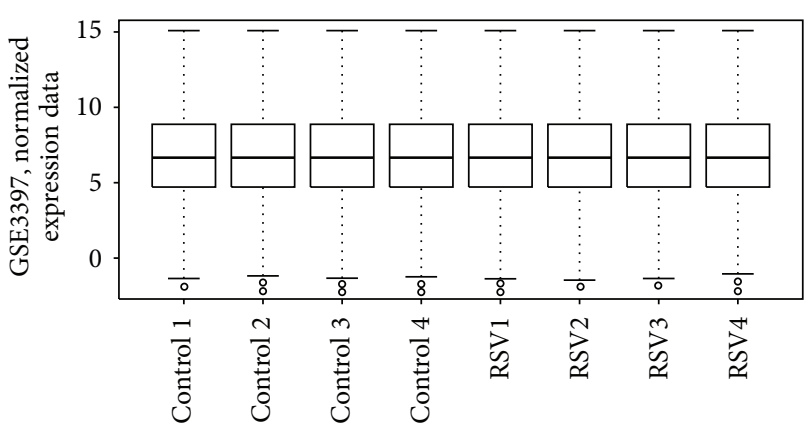

(b)

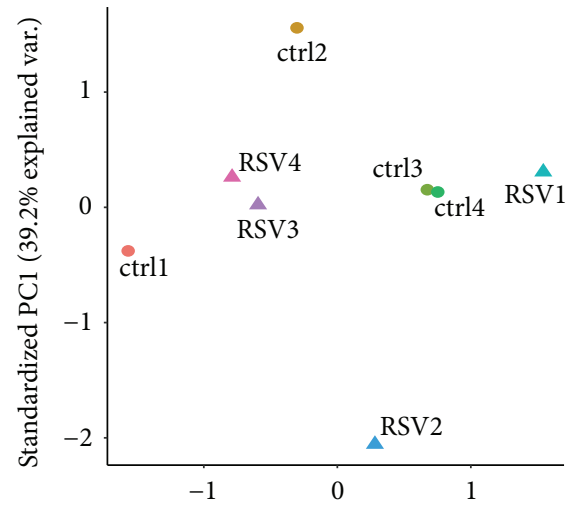

Standardized PC2 (21.9\% explained var.)

(e)

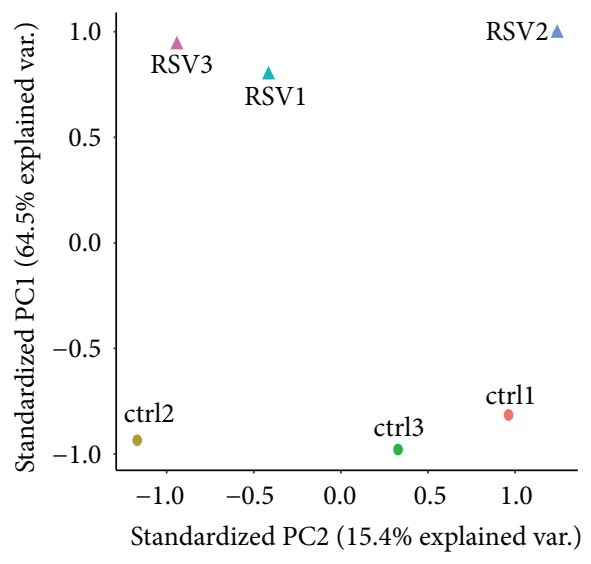

(h)

FIGURE 1: Preprocessing analysis of GEO datasets GSE3397 and GSE6802. (a) Boxplot of GSE3397, raw expression data. (b) Boxplot of GSE3397, normalized expression data. (c) Principal component analysis of GSE3397, raw expression data. (d) Principal component analysis of GSE3397, normalized expression data. (e) Principal component analysis of GSE3397, normalized and batch corrected expression data. (f) Boxplot of GSE6802, RMA normalized expression data. (g) Principal component analysis of GSE6802, normalized expression data. (h) Principal component analysis of GSE6802, $\log _{2}$ transformed RMA normalized expression data.

3.3. Functional Analysis. To obtain a biological interpretation of the transcriptional signature of RSV-infected BEAS-2B cells and compare with those reported by previous studies, enrichment analysis was performed with the online platform for functional analysis InnateDB [21]. Based on a FDR < 0.1, DEGs identified for GSE3397 were enriched in pathways related to Chromatin organization, histone acetylation, signaling by NOTCH, IL1, Integrin-linked kinase signaling, 


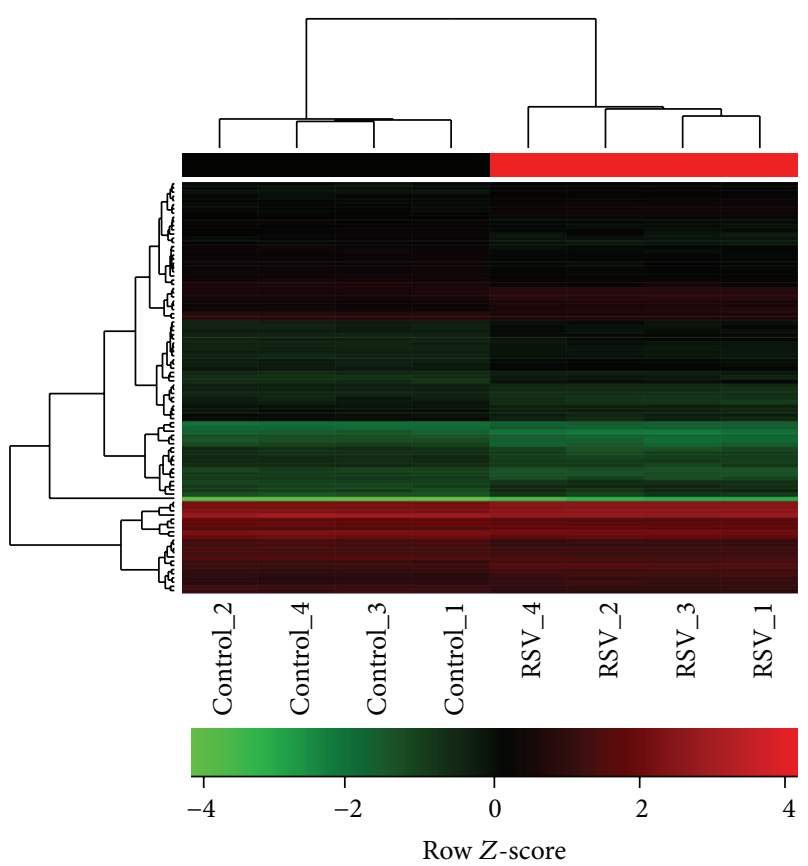

(a)

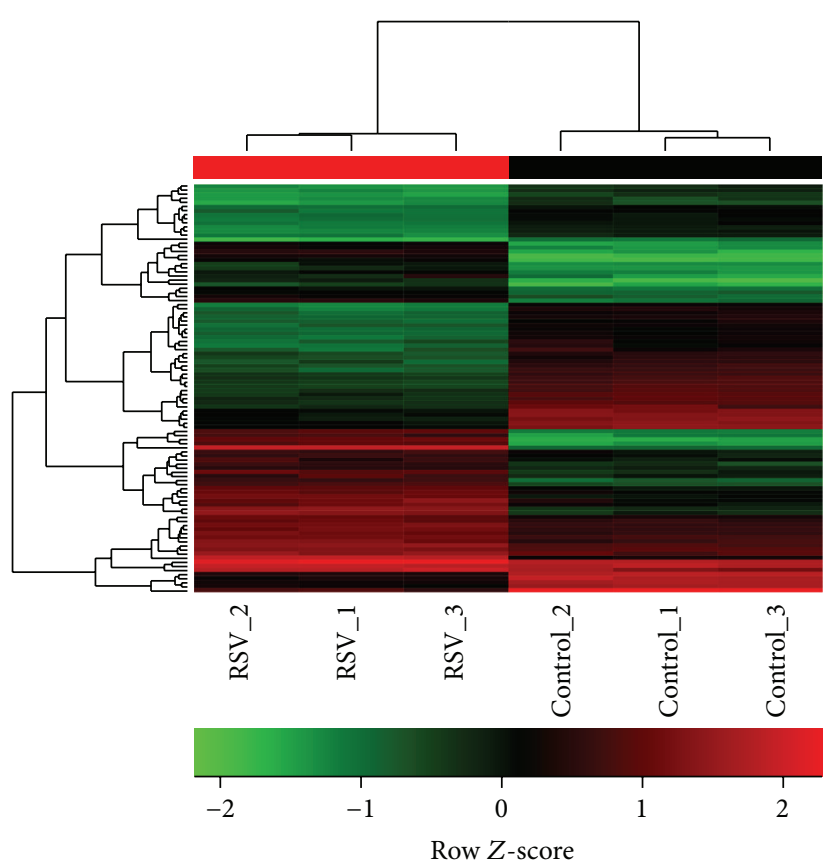

(b)

FIGURE 2: Transcriptional profiles of BEAS-2B cells infected with RSV for $4 \mathrm{~h}$. (a) Hierarchical clustering of differentially expressed genes from dataset GSE3397. (b) Hierarchical clustering of differentially expressed genes from dataset GSE6802. Row Z-scores were calculated based on normalized expression data. The colors from green to red represent the transition of decreased to increased expression.

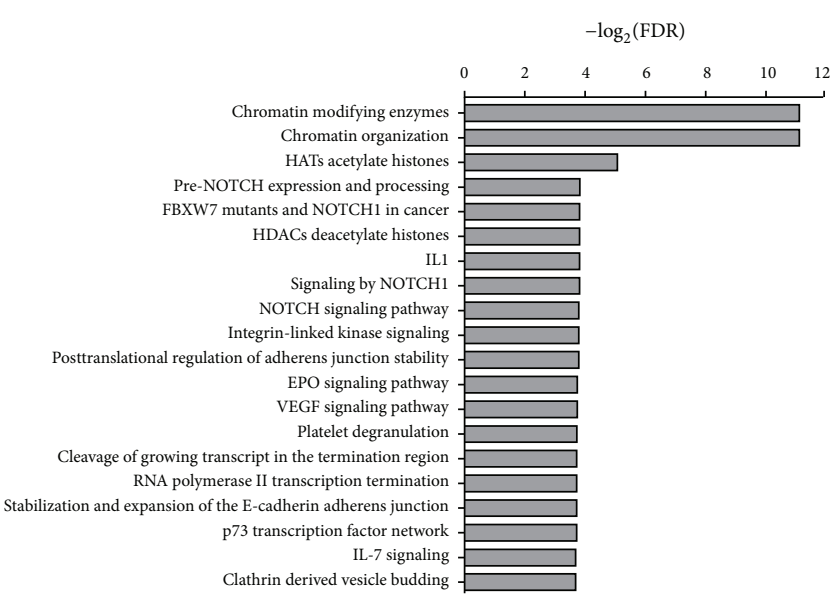

(a)

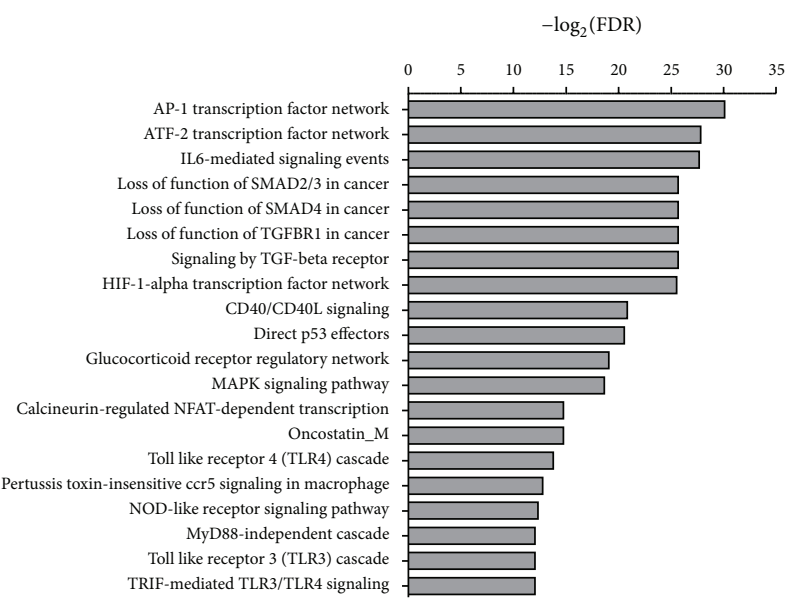

(b)

FIGURE 3: Pathway enrichment analysis with InnateDB. Differentially expressed genes from (a) GSE3397 or (b) GSE6802 were evaluated for overrepresentation in pathways annotated in databases as INOH, KEGG, NETPATH, PID NIC, and REACTOME.

EPO signaling pathway, VEGF signaling pathway, platelet degranulation, p73 transcription factor network, IL-7 signaling, p53 signaling pathway, and others (Figure 3(a) and Supplementary Data 1). Of interest, Huang and collaborators (2008) [13] reported gene overrepresentation within p53 signaling pathway, but only after $24 \mathrm{~h}$ following RSV infection of BEAS-2B cells. After $4 \mathrm{~h}$ following RSV infection, Huang and collaborators (2008) [13] only found a significant association with neuroactive ligand-receptor interaction pathway, which was not overrepresented in the present analysis. In contrast, DEGs resultant from dataset GSE6802 were enriched in pathways related to AP-1 transcription factor, ATF-2 transcription factor, IL-6 signaling, SMAD function, signaling by TGFBR, HIF- $1 \alpha$ transcription factor, signaling by CD40/CD40L, signaling by MAPK, signaling by innate immune receptors, and others (Figure 3(b) and Supplementary Data 1). Some of those pathways as CD40 signaling are indeed commonly induced by a variety of viral respiratory infections [22], whereas several of those pathways could indicate novel directions for studying the host response 


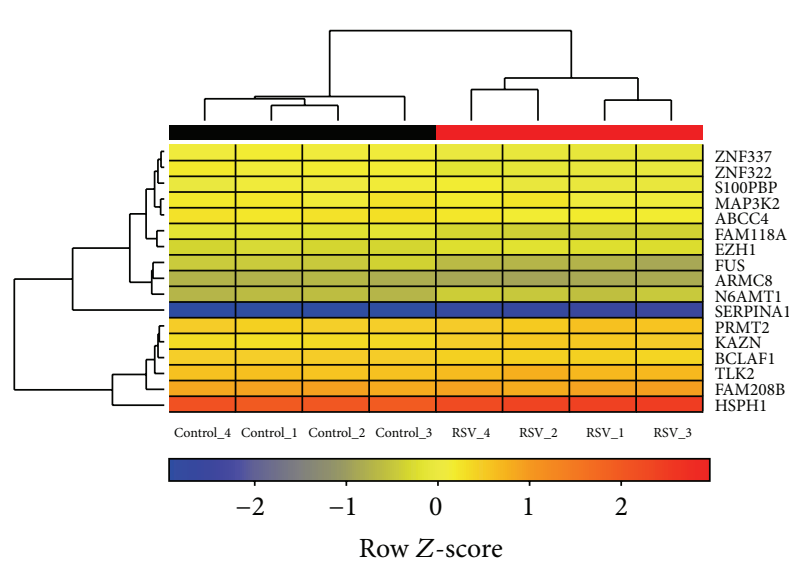

(a)

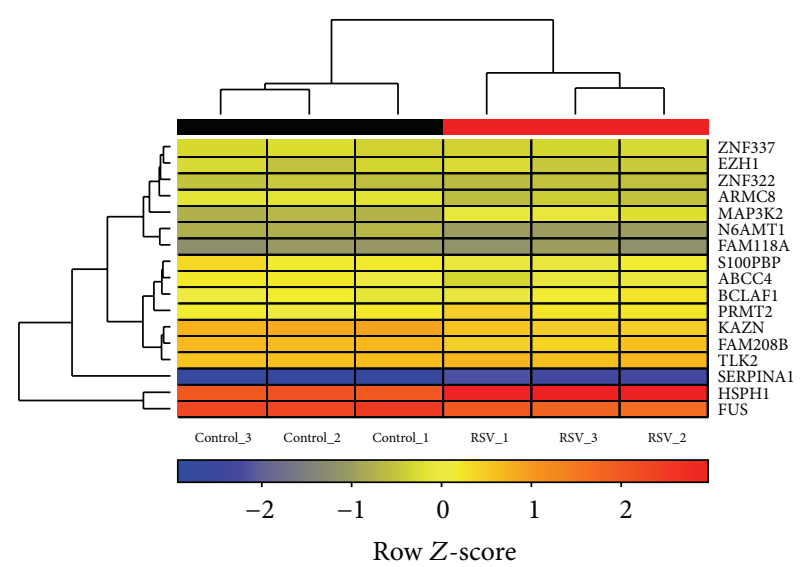

(b)

FIGURE 4: Biomarker signature of BEAS-2B cells infected with RSV for $4 \mathrm{~h}$. Hierarchical clustering of expression data for $A B C C 4, A R M C 8$, BCLAF1, EZH1, FAM118A, FAM208B, FUS, HSPH1, KAZN, MAP3K2, N6AMT1, PRMT2, S100PBP, SERPINA1, TLK2, ZNF322, and ZNF337 from (a) dataset GSE3397 and (b) dataset GSE6802. Row $Z$-scores were calculated based on normalized expression data. The colors from blue to red represent the transition of decreased to increased expression.

against RSV. Six pathways were enriched by DEGs from both datasets, the EPO signaling pathway, FBXW7 Mutants and NOTCH1 in Cancer, IL1, p53 signaling pathway, p73 transcription factor network, and signaling by NOTCH1. The erythropoietin (EPO) gene is a primary target of HIF-1 $\alpha$ transcription factor, whereas binding of HIF- $1 \alpha$ to the EPO enhancer promoter region induces transcriptional programs that influence inflammation and infection processes [23]. In addition, expression of Dll4, a major NOTCH ligand, is upregulated in dendritic cells infected with RSV, whereas blockage of Dll4 in vivo increased hyperreactivity of airways and mucus secretion that impacted the pathology of the disease, showing a key role of signaling by NOTCH in the regulation of immunity against RSV [24]. Moreover, besides modulations of the $\mathrm{p} 53$ signaling pathway by infection of RSV in vitro $[10,13]$, this pathway was found to be upregulated in whole blood of children with lower respiratory tract infection by RSV [25]. Taken together, those data point to key pathways which can impact infections of human bronchial epithelial cells with RSV.

\subsection{Meta-Analysis Based Biomarker Signature of RSV-Infected} $B E A S-2 B$ Cells. To determine a unique transcriptional signature of BEAS-2B cells induced by early infection with RSV, common DEGs for both datasets were further identified. The analysis retrieved a list of seventeen common genes: ABCC4, ARMC8, BCLAF1, EZH1, FAM118A, FAM208B, FUS, HSPH1, KAZN, MAP3K2, N6AMT1, PRMT2, S100PBP, SERPINA1, TLK2, ZNF322, and ZNF337 (Figure 4). Despite particular features in expression data from both datasets, unsupervised hierarchical clustering analysis based on this signature revealed the formation of robust clusters between RSV-infected or uninfected BEAS-2B cells (Figure 4). Of note, human airway epithelial cells were shown to express ABCC4/MRP4, a transporter for uric acid and cAMP [26].
Mucosal production of uric acid was recently linked to particulate matter-induced allergic sensitization [26]; therefore RSV infection could trigger such a response and contribute to the development and severity of allergic responses to particulate matter [27]. Moreover, both ABCC4 and SERPINA1 are annotated into the platelet degranulation pathway (Figure 3(a)), suggesting a role in antiviral mechanisms from bronchial epithelial cells. After an initial encounter with RSV, the transcriptional activity of human bronchial epithelial cells is reprogrammed to counteract viruses and other pathogens [10], whereas MAP3K2 and ZNF322 are clearly involved on the activation and regulation of MAP kinase signaling pathway $[28,29]$. Indeed, RSV infection leads to the activation of p38 MAPK [30] and c-JUN kinase pathway, which negatively regulates the production of TNF$\alpha$ in human epithelial cells [31] and might contribute to virus evasion from an early immune response. Interestingly, the biosignature also included BCLAF1, a molecule involved in processes as apoptosis, transcription and processing of RNA, and export of mRNA from the nucleus [32]. However, this nuclear protein was also implicated as a viral restriction factor targeted to degradation by human cytomegalovirus [32]. Moreover, EZH1 was shown to be involved in the methylation of histone 3 at lysine 27 (H3K27) of the HIV provirus in resting cells [33] and could thus exert a significant function in infections with RSV, whereby other genes such as N6AMT1, FUS, and PRMT2 are also involved in protein methylation. Indeed, using coimmunoprecipitation and mass spectrometry, recent work demonstrated that RSV nucleoprotein $(\mathrm{N})$ interacts with protein arginine $\mathrm{N}$ methyltransferase 5 (PRMT5) [34], suggesting that PRMT2 could also interact with RSV proteins and play an important role during infections of human bronchial epithelial cells. Several of the genes identified in this study have been poorly studied in the context of RSV infection, whereby none of 
them was previously reported as a biomarker of infections by this virus. Of note, except for FAM208B and $K A Z N$, analysis conducted by Smith and collaborators (2012) [22] which included both datasets (GSE3397 and GSE6802) also identified the significant modulation of the genes included in the biomarker signature identified herein.

\section{Conclusions}

The combined analysis of distinct datasets from BEAS-2B cells infected with RSV retrieved intriguing results, whereby using powerful statistical methods and assumptions this study identified a new set of biomarkers of early infection with RSV composed by seventeen genes: ABCC4, ARMC8, BCLAF1, EZH1, FAM118A, FAM208B, FUS, HSPH1, KAZN, MAP3K2, N6AMT1, PRMT2, S100PBP, SERPINA1, TLK2, ZNF322, and ZNF337. This transcriptional signature could be useful for the development of molecular diagnosis tools as well as future investigations of processes involved in hostpathogen interactions.

\section{Competing Interests}

The author declares that there are no competing interests regarding the publication of this paper.

\section{Acknowledgments}

The author is grateful to Dr. Fátima Pereira de Souza for critical comments on the paper. Luiz Gustavo Gardinassi was supported by scholarships from Fundação de Amparo à Pesquisa do Estado de São Paulo (FAPESP).

\section{References}

[1] L. J. Anderson, R. A. Parker, and R. L. Strikas, "Association between respiratory syncytial virus outbreaks and lower respiratory tract deaths of infants and young children," The Journal of Infectious Diseases, vol. 161, no. 4, pp. 640-646, 1990.

[2] A. R. Falsey and E. E. Walsh, "Respiratory syncytial virus infection in adults," Clinical Microbiology Reviews, vol. 13, no. 3, pp. 371-384, 2000.

[3] V. F. Botosso, P. M. D. Zanotto, M. Ueda et al., "Positive selection results in frequent reversible amino acid replacements in the G protein gene of human respiratory syncytial virus," PLoS Pathogens, vol. 5, no. 1, Article ID e1000254, 2009.

[4] L. G. A. Gardinassi, P. V. M. Simas, D. E. Gomes et al., "Diversity and adaptation of human respiratory syncytial virus genotypes circulating in two distinct communities: public hospital and day care center," Viruses, vol. 4, no. 11, pp. 2432-2447, 2012.

[5] L. G. Gardinassi, P. V. Marques Simas, J. B. Salomão et al., "Seasonality of viral respiratory infections in southeast of Brazil: the influence of temperature and air humidity," Brazilian Journal of Microbiology, vol. 43, no. 1, pp. 98-108, 2012.

[6] R. A. Tripp, "Respiratory syncytial virus (RSV) modulation at the virus-host interface affects immune outcome and disease pathogenesis," Immune Network, vol. 13, no. 5, pp. 163-167, 2013.

[7] T. H. Mogensen, "Pathogen recognition and inflammatory signaling in innate immune defenses," Clinical Microbiology Reviews, vol. 22, no. 2, pp. 240-273, 2009.
[8] G. Diamond, D. Legarda, and L. K. Ryan, "The innate immune response of the respiratory epithelium," Immunological Reviews, vol. 173, pp. 27-38, 2000.

[9] R. J. Boyton and P. J. Openshaw, "Pulmonary defences to acute respiratory infection," British Medical Bulletin, vol. 61, pp. 1-12, 2002.

[10] A. K. Mayer, M. Muehmer, J. Mages et al., "Differential recognition of TLR-dependent microbial ligands in human bronchial epithelial cells," Journal of Immunology, vol. 178, no. 5, pp. 31343142, 2007.

[11] I. Martínez, L. Lombardía, B. García-Barreno, O. Domínguez, and J. A. Melero, "Distinct gene subsets are induced at different time points after human respiratory syncytial virus infection of A549 cells," Journal of General Virology, vol. 88, no. 2, pp. 570581, 2007.

[12] Y. Zhang, B. A. Luxon, A. Casola, R. P. Garofalo, M. Jamaluddin, and A. R. Brasier, "Expression of respiratory syncytial virusinduced chemokine gene networks in lower airway epithelial cells revealed by cDNA microarrays," Journal of Virology, vol. 75, no. 19, pp. 9044-9058, 2001.

[13] Y.-C. T. Huang, Z. Li, X. Hyseni et al., "Identification of gene biomarkers for respiratory syncytial virus infection in a bronchial epithelial cell line," Genomic Medicine, vol. 2, no. 3-4, pp. 113-125, 2008.

[14] R. Ihaka and R. Gentleman, "R: a language for data analysis and graphics," Journal of Computational and Graphical Statistics, vol. 5, no. 3, pp. 299-314, 1996.

[15] R. C. Gentleman, V. J. Carey, D. M. Bates et al., "Bioconductor: open software development for computational biology and bioinformatics," Genome Biology, vol. 5, no. 10, article R80, 2004.

[16] L. Gautier, L. Cope, B. M. Bolstad, and R. A. Irizarry, "Affyanalysis of Affymetrix GeneChip data at the probe level," Bioinformatics, vol. 20, no. 3, pp. 307-315, 2004.

[17] W. E. Johnson, C. Li, and A. Rabinovic, "Adjusting batch effects in microarray expression data using empirical Bayes methods," Biostatistics, vol. 8, no. 1, pp. 118-127, 2007.

[18] J. T. Leek, W. E. Johnson, H. S. Parker, A. E. Jaffe, and J. D. Storey, "The SVA package for removing batch effects and other unwanted variation in high-throughput experiments," Bioinformatics, vol. 28, no. 6, Article ID bts034, pp. 882-883, 2012.

[19] M. E. Ritchie, B. Phipson, D. Wu et al., "Limma powers differential expression analyses for RNA-sequencing and microarray studies," Nucleic Acids Research, vol. 43, article e47, 2015.

[20] G. R. Warnes, B. Bolker, L. Bonebakker et al., "gplots: various R programming tools for plotting data," R Package Version, vol. 2, no. $4,2009$.

[21] K. Breuer, A. K. Foroushani, M. R. Laird et al., "InnateDB: Systems biology of innate immunity and beyond-recent updates and continuing curation," Nucleic Acids Research, vol. 41, no. 1, pp. D1228-D1233, 2013.

[22] S. B. Smith, W. Dampier, A. Tozeren, J. R. Brown, and M. MagidSlav, "Identification of common biological pathways and drug targets across multiple respiratory viruses based on human host gene expression analysis," PLoS ONE, vol. 7, no. 3, Article ID e33174, 2012.

[23] M. V. Sitkovsky, D. Lukashev, S. Apasov et al., "Physiological control of immune response and inflammatory tissue damage by hypoxia-inducible factors and adenosine A2A receptors," Annual Review of Immunology, vol. 22, pp. 657-682, 2004. 
[24] M. A. Schaller, R. Neupane, B. D. Rudd et al., "Notch ligand Delta-like 4 regulates disease pathogenesis during respiratory viral infections by modulating Th2 cytokines," The Journal of Experimental Medicine, vol. 204, no. 12, pp. 2925-2934, 2007.

[25] A. Mejias, B. Dimo, N. M. Suarez et al., "Whole blood gene expression profiles to assess pathogenesis and disease severity in infants with respiratory syncytial virus infection," PLOS Medicine, vol. 10, no. 11, Article ID e1001549, 2013.

[26] M. J. Gold, P. R. Hiebert, H. Y. Park et al., "Mucosal production of uric acid by airway epithelial cells contributes to particulate matter-induced allergic sensitization," Mucosal Immunology, vol. 9, pp. 809-820, 2016.

[27] M. Barends, A. Boelen, L. de Rond et al., "Influence of respiratory syncytial virus infection on cytokine and inflammatory responses in allergic mice," Clinical and Experimental Allergy, vol. 32, no. 3, pp. 463-471, 2002.

[28] Y. Li, Y. Wang, C. Zhang et al., "ZNF322, a novel human $\mathrm{C}_{2} \mathrm{H}_{2}$ Krüppel-like zinc-finger protein, regulates transcriptional activation in MAPK signaling pathways," Biochemical and Biophysical Research Communications, vol. 325, no. 4, pp. 13831392, 2004.

[29] P. K. Mazur, N. Reynoird, P. Khatri et al., "SMYD3 links lysine methylation of MAP3K2 to Ras-driven cancer," Nature, vol. 510, no. 7504, pp. 283-287, 2014.

[30] T. R. Meusel and F. Imani, "Viral induction of inflammatory cytokines in human epithelial cells follows a p38 mitogenactivated protein kinase-dependent but NF- $\kappa \mathrm{B}$-independent pathway," The Journal of Immunology, vol. 171, no. 7, pp. 37683774, 2003.

[31] M. J. Stewart, S. B. Kulkarni, T. R. Meusel, and F. Imani, "cJun N-terminal kinase negatively regulates dsRNA and RSV induction of tumor necrosis factor- $\alpha$ transcription in human epithelial cells," Journal of Interferon and Cytokine Research, vol. 26, no. 8, pp. 521-533, 2006.

[32] S. H. Lee, R. F. Kalejta, J. Kerry et al., "BclAF1 restriction factor is neutralized by proteasomal degradation and microRNA repression during human cytomegalovirus infection," Proceedings of the National Academy of Sciences of the United States of America, vol. 109, no. 24, pp. 9575-9580, 2012.

[33] M. K. Tripathy, M. E. M. McManamy, B. D. Burch, N. M. Archin, and D. M. Margolis, "H3K27 demethylation at the proviral promoter sensitizes latent HIV to the effects of vorinostat in ex vivo cultures of resting CD $4^{+} \mathrm{T}$ cells," Journal of Virology, vol. 89, no. 16, pp. 8392-8405, 2015.

[34] A. P. Oliveira, F. M. Simabuco, R. E. Tamura et al., "Human respiratory syncytial virus $\mathrm{N}, \mathrm{P}$ and $\mathrm{M}$ protein interactions in HEK-293T cells," Virus Research, vol. 177, no. 1, pp. 108-112, 2013. 

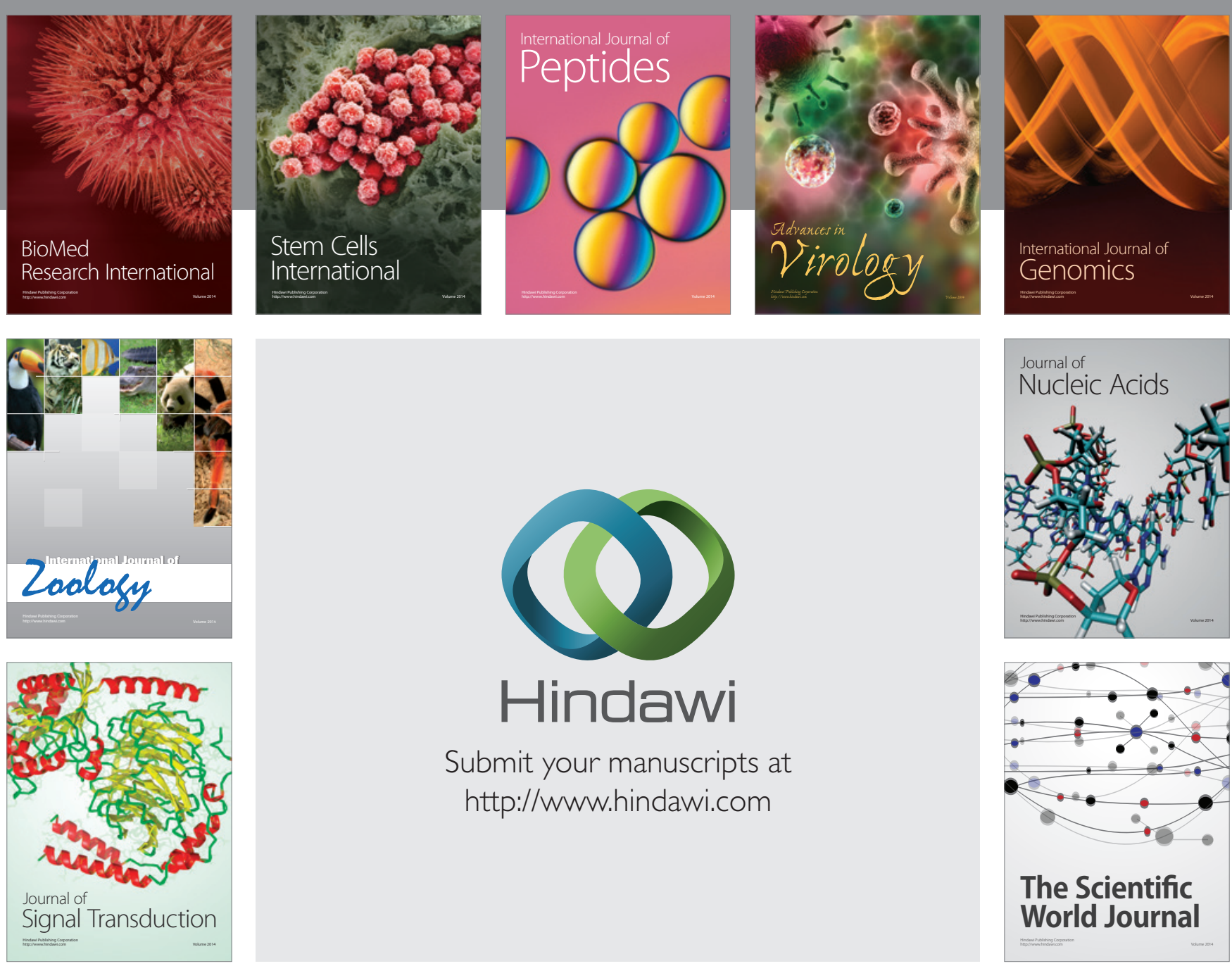

Submit your manuscripts at

http://www.hindawi.com
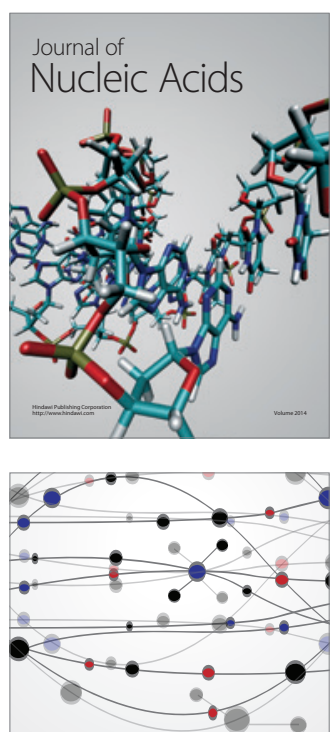

The Scientific World Journal
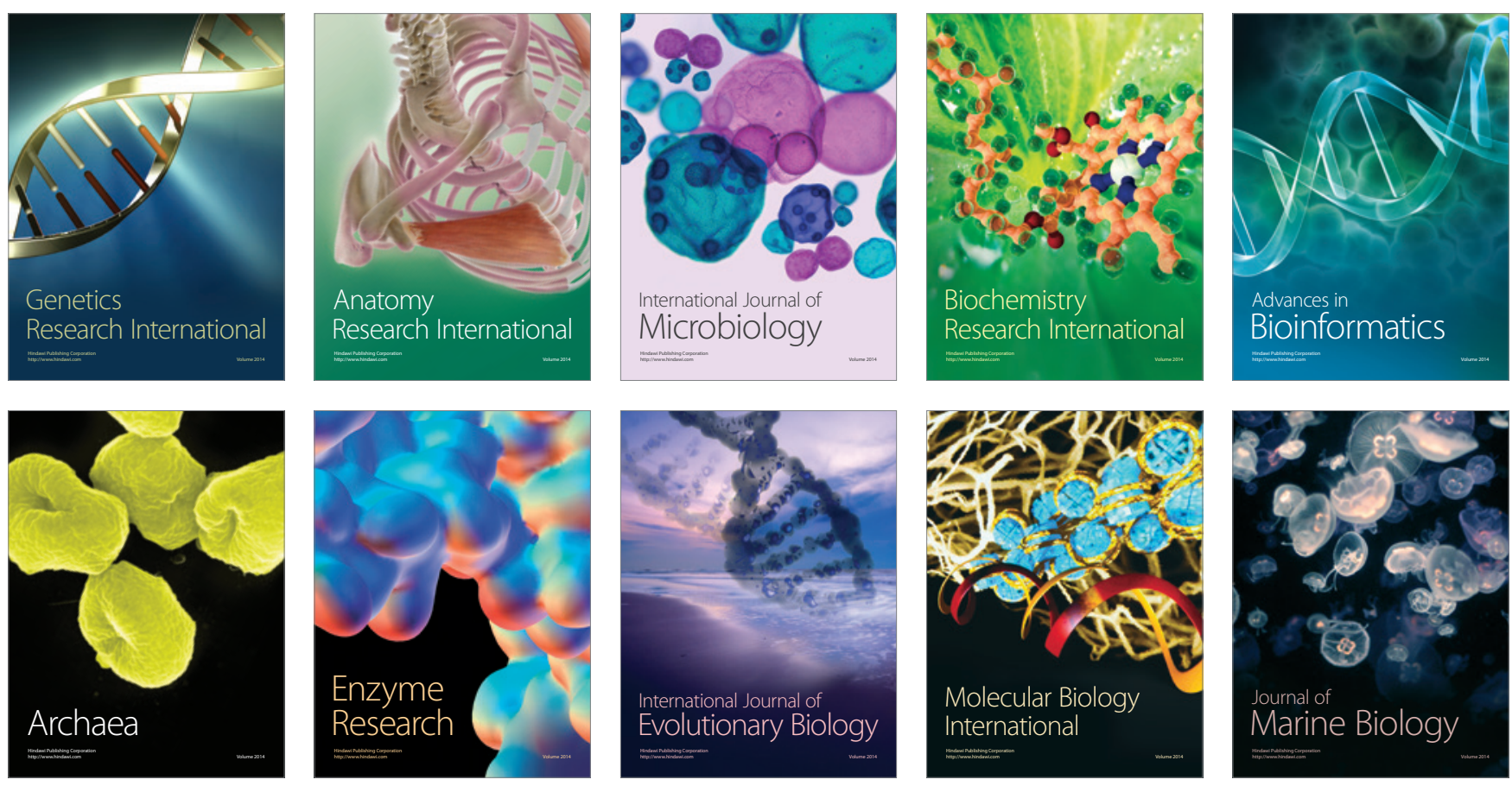